\title{
Geriatric infections: Decreased immunity or evolved opportunists?
}

\author{
Ulfat Baig $^{1}$, Vidhya Laxmi ${ }^{1}$, Akanksha Ojha ${ }^{l}$ and Milind Watve ${ }^{2}$ (1) \\ ${ }^{1}$ Indian Institute of Science Education and Research, Dr. Homi Bhabha Road, Pune 411 008, India \\ ${ }^{2}$ Deenanath Mangeshkar Hospital and Research Centre, Erandawne, Pune 411 004, India \\ *Corresponding author (Email, milind.watve@gmail.com)
}

MS received 9 June 2018; accepted 2 March 2020; published online 10 April 2020

\begin{abstract}
In host-parasite co-evolution, parasites are assumed to have an advantage owing to their shorter generation time. Evolution of pathogens within the lifetime of a host individual is implicated as a strong selective force in the evolution of sex and aging in the host. However, this assumption or its testable predictions have not been examined empirically. We classified infectious bacteria and viruses into those that can have continued longterm existence on the host body (group 1) versus those that have only a short-term interaction during an active infection (group 2). We surveyed the literature for age-specific incidence data about infections from both the groups. The age trends of the two groups show contrasting patterns. The incidence of infections by all group 1 pathogens showed a 2.28- to 28-fold increase in older ages. In group 2, 6 out of the 9 pathogens showed a significant declining trend in incidence with age. In both groups, there was greater mortality or morbidity among the infected in the old-age classes. These patterns are better explained by pathogen evolution than by age-related decline in immunity.
\end{abstract}

Keywords. Geriatric infections; host-parasite co-evolution; immunity; microbiota; pathogens

\section{Introduction}

The incidence of a number of infections appears to increase in old age (Yoshikawa 2000; Schneider 1983; Gardner 1980; Yoshikawa and Norman 2017; Bender 2003; Meyers 1989). There are three possible causes for this pattern. Although there is substantial literature on immunosenescence in several species of animals (Papp et al. 2012; Sidler et al. 2013; Youngman et al. 2011; Zerofsky et al. 2005), the assertion that immunosenescence forms the foundation for infectious disease in elderly humans, according to Schneider (1983), '...lacks a firm scientific foundation'. Although over the last four decades, substantial literature on the molecular and cellular aspects of immunosenescence has been added, many fundamental questions about the evolution of these mechanisms, its relevance to aging and health, even a precise definition of immunosenes- cence are under substantial haze and debate (Fulop et al. 2018; Pawelec 2018). Alternatively, it is possible that a decline in the overall physiological capacity increases morbidity and mortality at the same level of infection. The third possible cause, largely overlooked by medicine, is that the opportunistic pathogens associated with the body evolve within the lifetime of an individual to overcome its resistance mechanisms. Evolutionary biologists have recognized this imbalance in the host-parasite co-evolution. Pathogens have an upper hand in the host-parasite co-evolution owing to their faster rate of evolution. Pathogen evolution within the lifetime of an individual is recognized to play a role in the evolution of sex (Hamilton 1980) and the evolution of host aging (Ulfat et al. 2017).

The three hypotheses are not mutually exclusive and it is difficult to quantify their relative importance. Nevertheless, it is possible to make and test specific

Electronic supplementary material: The online version of this article (https://doi.org/10.1007/s12038-020-0025-x) contains supplementary material, which is available to authorized users. 
differential predictions from the different causal hypotheses. At least under certain conditions, these predictions allow us to differentiate between the competing hypotheses:

(i) If decline in general immunity is the main cause, the incidence of all types of infections is expected to increase in old age although not to the same extent. The body is exposed to opportunistic pathogens more often than to externally acquired infections. Therefore, the incidence of opportunistic infections is expected to increase substantially more than that by externally acquired pathogens. However, since there are alternative explanations for an increase in opportunistic infections (hypothesis (iii) stated below), they cannot be taken to support this hypothesis. A significant increase in the incidence of externally acquired infections is required to support this hypothesis. (ii) Agerelated physiological deterioration is expected to increase the morbidity and mortality among the infected population. Alternatively, a significantly greater increase in the morbidity-mortality in comparison with the increase in incidence can be taken as a support to this hypothesis. (iii) If evolution of opportunistic pathogens is the major cause, the incidence of opportunistic infections should specifically increase in old age without affecting the incidence of externally acquired infections.

It is likely that all three play a role in geriatric infections and it may not be possible to resolve between the three hypotheses under every condition. Nevertheless, an exploration into the competing hypotheses and an attempt to falsify one or more of them can certainly be attempted. We searched the literature on age-related trends in incidence to see whether there are consistent trends across studies and whether one or more of the above hypotheses can be falsified or supported using them.

\section{Methodology}

\subsection{Choice of pathogens and inclusion-exclusion criteria}

In order to compare the age specific incidence of opportunistic versus non-opportunistic infections, we selected pathogens that are sufficiently common globally, for whom there is good background epidemiological information including whether and with what frequency they inhabit the host body under normal conditions, whether a long-term carrier state is known, and whether they confer long-term immunity. Known childhood infections, sexually transmitted infections, zoonotic and vector borne infections were excluded. The infectious agents selected were divided in to two groups (table 1): group 1 comprising pathogens that can exist on or within a host body for prolonged period either as a part of normal microbiota, long-term carrier state or silent subclinical existence; and group 2 comprising pathogens that do not constitute the normal body flora and do not have long-term (1e year being used as a cutoff here) association with individual host bodies.

Pathogens from group 1 have the opportunity to evolve to overcome the specific defence mechanisms of an individual host, whereas group 2 pathogens do not have this opportunity owing to a short-term association with any host individual.

\subsection{Data search and sources}

We searched PubMed and Google Scholar for individual published reports giving age-class-specific incidence, in addition to available online data from Centers for Disease Control and Prevention; The National Epidemiological Surveillance of Infectious Diseases; Williamson County \& Cities Health District, Kentucky; Public Health Agency of Canada; Canada Communicable Disease Report; National Surveillance Programme for Communicable Diseases; Cabinet for Health and Family Services; Indiana State Department of Health; Louisiana Department of Health and Hospitals - Office of Public Health; Census India; Pakistan Bureau of Statistics, and The Bangladesh

Table 1. The list of pathogens considered for the analysis following the inclusion and exclusion criteria

\begin{tabular}{rll}
\hline & \multicolumn{1}{c}{ Group 1 } & \multicolumn{1}{c}{ Group 2 } \\
\hline 1. & Pneumococcus & Shigella \\
2. & Staphylococcus & Vibrio \\
3. & Group A Streptococcus & Coronavirus \\
4. & Group B Streptococcus & Nocardia \\
5. & E. coli & Leptospira \\
6. & Helicobacter & Non-typhoid Salmonella \\
7. & Neisseria & Mycoplasma \\
8. & Clostridium & Influenza \\
9. & Enterococcus & Typhoid \\
10. & M. tuberculosis & \\
11. & Pseudomonas & \\
\hline
\end{tabular}

The 20 pathogens were divided according to the time of association with the individual host (Boyd 1995; Chakraborty 2005; Dey and Dey 1973; Goering et al. 2012; Ryan et al. 2014; Tortora et al. 2013). 
Bureau of Statistics. For infections such as urinary tract infections that can be caused by many different pathogens, only studies with confirmed identification of pathogens were included (supplementary table 1). For many pathogens more than one datasets were available. Where the reported age classes matched and the age trends were comparable, we pooled the data. Incidence data were pooled only for looking at the quantitative trends (figure 1). Wherever the reported age classes were different or the trends were contradicting, we did not pool the data but plotted them separately. For significance testing, the data were not pooled but individual trends were tested separately using the selected statistical methods (see below). The systematic review design followed Preferred Reporting Items for Systematic Reviews and Meta-analyses guidelines.

\subsection{Statistical analysis}

Age-specific incidence of clinically diagnosed infections for each of the selected pathogens was calculated as the number of cases per 100,000 age-group-specific population. Data could be used as reported from papers in which the age-normalized incidence was given.
Wherever the reported data was in terms of the number of cases, age normalization was done by accessing age structure demographic data of the specific country (Bangladesh Bureau of Stastitics, 2017; Pakistan Bureau of Statistics, 2017; Directorate of Census Operation, Tamil Nadu, 2017). Alternatively, we requested unreported demographic data from authors wherever required. The age grouping in different data sources was often different and could not be matched exactly.

In order to enable a comparative analysis, we considered the incidence in the age group covering the age 20 as unit incidence, and incidence in all other age groups was expressed as multiples of this unit. This normalization allowed comparison across pathogens with widely differing incidences. The significance of the trend in each of the individual datasets was tested using non-parametric correlations since the trends were typically nonlinear. Data on the same population but at different years were considered as different sets. If male/female incidence was reported separately, they were analysed separately. We avoided the childhood data (age <20) in analysing trends so as to avoid complications due to childhood immunity. The consistency in the age trend was analysed using Spearman's Rho correlation using Excel 2007 and Prism 5.
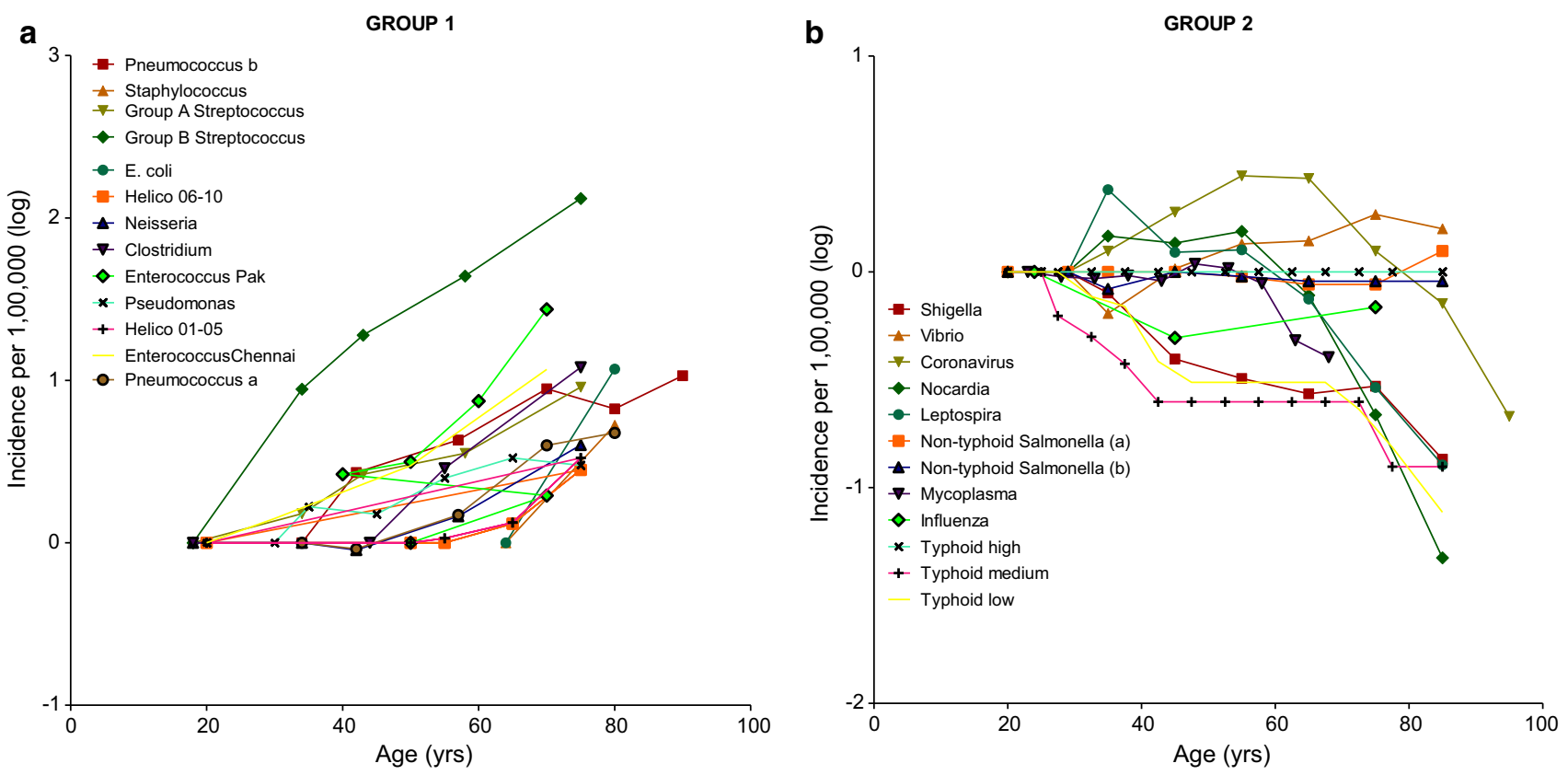

Figure 1. Normalized age-specific incidence trends of 20 pathogens: Group 1 pathogens (a) that have an opportunity to evolve on the body of individual hosts show an increase with age. Group 2 pathogens (b) that have short-term associations with the host body and therefore little opportunity to evolve within the lifetime of host individuals, do not show an increase in incidence. In fact, many show a significant negative trend with age. Two pathogens, namely $M$. tuberculosis and $V$. cholera, showed mixed trends and are not included in the figure. 
In addition to considering the significance of individual Rho at the significance level of 0.05 , we also looked at how many datasets had positive or negative Rho values to test consistency in the trends across datasets. This was particularly important since in some datasets: only two age classes were recognized, and so individual Rho calculations were meaningless. For example, in opportunistic E. coli infections the data by Flasche et al. (2011) had only two age groups but there were 5 such datasets and all of them had an increasing trend. Therefore, although we could not test individual trends for significance, the consistency across datasets was tested to be non-random by a chi square test. At the third level, we also looked at the total number of negative and positive trends in group 1 versus group 2 pathogens. This analysis, being non-parametric, does not give a quantitative idea of the fold increase in incidence with age. The fold change in incidence in the pooled data was represented in age trend diagram (figure 1) and calculated as the ratio of the incidence per 100,000 in the age class over $60 \mathrm{~s}$ to the incidence in the age class covering the age 20 .

For testing the physiological decline hypothesis, we looked for publications reporting age-specific mortality rates among the infected individuals. Alternatively, we compared age trends in the population mortality with those in incidence wherever available.

\section{Results}

According to the inclusion and exclusion criteria, we obtained 176 datasets from 20 pathogen species. The monotonicity of trends of incidences with age groups in every individual dataset was tested using non-parametric correlations (supplementary table 2). Trends in the age-specific incidence clearly fell into two types of patterns (figure 1). In one, consisting of 10 species of pathogens and 14 pooled datasets, the incidence invariably increased with age. Between the age groups covering the $20 \mathrm{~s}$ and over- $60 \mathrm{~s}$, the increase was remarkable ranging from 2.28 -fold to 28 -fold. In the other pattern followed by 8 species and 12 pooled datasets, there was a significant negative trend in 6 with a $50 \%$ to $95 \%$ decrease in incidence between the 20 and over-60 age groups. The two patterns corresponded very closely with the two groups in table 1. All pathogens of group 1 showed a positive incidence trend with age. For two datasets, data on only two age groups relevant to our question was available. Here we could not perform any statistical analysis for the trend in individual datasets, but in both the groups the incidence was higher in the higher age class in every dataset examined.

The statistical significance of the trends was tested non-parametrically. The Spearman's Rho is a good indicator of monotonic trends even if they are nonlinear. For all datasets that had more than three adult age groups, we tested the significance of individual trends. In group 1 pathogens, 116 out of 118 trends were positive, out of which 88 were individually significant. Only two Rho values were negative and they were not significant. For every group 1 pathogen, either individual positive trends or the consistency across trends was significant. Out of 58 datasets for group 2 pathogens, 46 trends were negative, 32 being individually significant. Out of the 12 positive trends, only 3 were individually significant. Out of 9 pathogens, 6 had significantly decreasing age incidence curves. Influenza had a mixed trend ( 8 negative and 7 positive Rho values), cholera had 1 significant negative and 4 nonsignificant positive Rho values, and coronavirus had 3 negative trends but none significant. Thus, collectively group 1 pathogens, having opportunity to evolve on the host body, had a predominantly increasing age incidence trend, and group 2 pathogens, not having such opportunity, had predominantly decreasing one. This can be best viewed collectively in the frequency distribution of all Rho values which lie on the positive extreme for group 1 pathogens and mostly on the negative side for group 2 (figure 2).

Although the incidence trends in group 2 pathogens were predominantly declining, we examined whether the trends in mortality and morbidity were also different. The specific case of influenza is demonstrated in figure 3, which shows that in contrast to the incidence trend, the hospitalization rate as well as mortality rate clearly increased with age (CDC Influneza Vaccine Activity, US, 2012-2014; Dávila et al. 2014; CDC Prevention and Control of Influenza with Vaccines, 2010). The contrast in the incidence and morbidity-mortality trends supports the physiological decline hypothesis more than the immunity decline hypothesis since the latter predicts rise in incidence as well.

\section{Discussion}

A remarkable consistency was observable in the age trends for any given pathogen. For any pathogen for which we had multiple sources of data, the trends were very similar across populations, gender and years, with the only possible exception of influenza and cholera. 


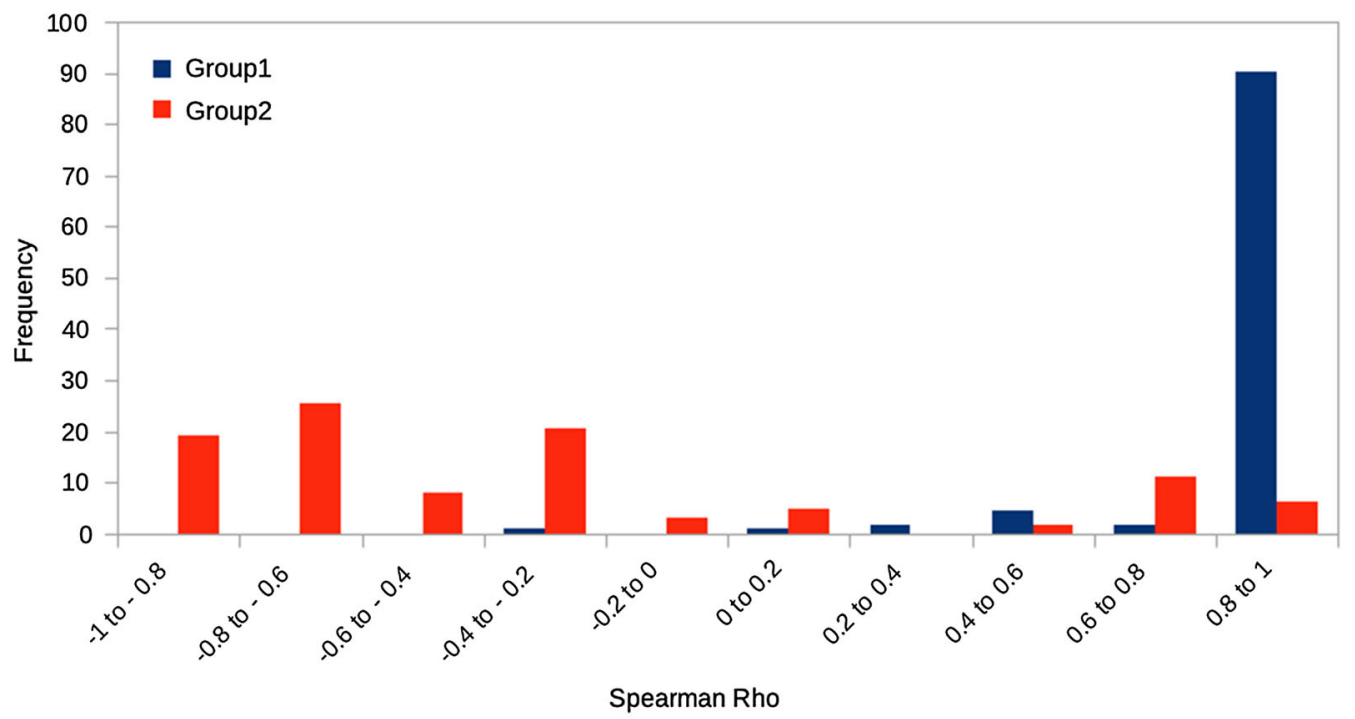

Figure 2. Frequency distribution of Spearman's Rho reflecting incidence trends with age. Note that for group 1 pathogens, the Rho values are predominantly close to +1 ; for group 2 they are mostly negative with a few exceptions.

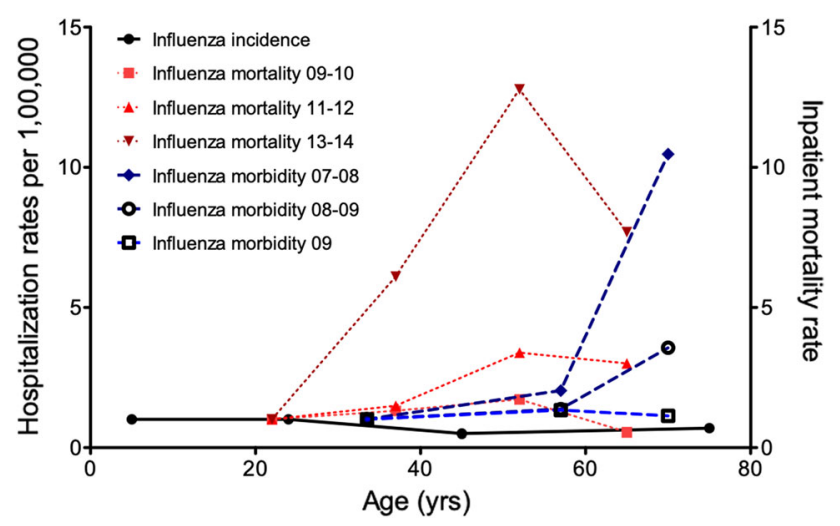

Figure 3. Comparison of age-wise change in incidence, hospitalization and mortality rates of diseases: Age-wise incidence rate, hospitalization rate and mortality for influenza from different sources (Dávila et al. 2014; Goodwin et al. 2006; CDC Influneza Vaccine Activity-US 2012-2014; CDC Prevention and Control of Influenza with Vaccines 2010). The data includes influenza-induced morbidity from the years 2007-2009 and influenza-caused mortality from 2009 to 2014 . In contrast to incidence rate, there is an increase in hospitalization and mortality rate with aging.

The predominant trends of these 20 pathogen groups are consistent with the pathogen evolution hypothesis. As an alternative to evolution on individual host body, one can possibly argue that some of the opportunistic pathogens induce immunosenescence in the host (Aguilera et al. 2018), and therefore their incidence increases with age. However, if they induce general immunosenescence, the host susceptibility to all kinds of infections should increase. The failure to increase the incidence of externally acquired pathogens makes this interpretation unlikely.

The prediction specific to the declining immunity hypothesis that the incidence of externally acquired infections should also increase with age failed to obtain generalized support in epidemiological data. This result needs to be interpreted carefully because age-related decline in immunity is a widely held belief in the field of medicine. Studies on individual components of immunity appear to support this hypothesis. For example, reduction in antibody response to vaccines in old age has been demonstrated (Goodwin et al. 2006). However, incidence patterns should be the ultimate marker of immunity to infections.

In clinical research, often pathway intermediates or surrogate markers are used for the risk of a disease, such as LDL cholesterol for cardiovascular disease. Although such markers make early inferences possible, the ultimate test is the real end point. In many drug trials, a reduction in LDL was not accompanied by a reduction in cardiovascular events (AIM-HIGH investigators 2011). Similarly, normalization of blood sugar failed to arrest complications of diabetes in many trials (NICE Trial 2008; ACCORD 2008). Similarly, it is likely that a decline in immunity with old age is apparent when looking at certain mechanisms or surrogate markers, but the ultimate test needs to be based on the actual incidence of disease.

Incidence of infection depends upon exposure to pathogen propagules and it might be argued that for certain infections exposure may decline with age. We excluded sexually transmitted infections and vector- 
borne infections where a change in exposure with age is more likely. For airborne pathogens, the exposure is expected to be more homogeneous in a population but we do not see any consistent age-related increase in such infections. Quantitatively, we see a 10- to 30-fold rise in the incidence of 5 to 6 group 1 pathogens, for equal number of group 2 pathogens, there is a decline up to one-tenth in the incidence (figure 1). If the difference in exposure was to account for it, a 100- to 300 -fold difference in exposure is required. In the absence of data on exposure level, this explanation remains weak although it might explain a small part of the trend. The epidemiological patterns do not falsify the immunosenescence hypothesis but show that immunosenescence is not strong enough to overpower the other age-related factors that might decrease the age-specific incidence.

In a nutshell, epidemiological analysis suggests that evolution of opportunistic pathogens to overcome resistance mechanisms of individual hosts is likely to be the major contributor to geriatric infections. Although decreased immunity can increase infections by opportunistic pathogens, it should also show at least some increase in externally acquired infections. The fact that there is a significant decrease in most group 2 infections undermines the generalization of declined immunity. While physiological deterioration appears to contribute to increased morbidity and mortality among the infected, the signature of generalized immunosenescence did not appear to be very strong in the epidemiological data.

Pathogens are rapidly evolving and the rate of evolution is orders of magnitude faster than that of the host. This imbalance in the host-parasite co-evolution is thought to be a major selective force in the evolution of sex and that of aging (Hamilton 1980; Ulfat et al. 2017). However, evolution of any member of the microbiota within the lifetime of an individual has not yet been demonstrated. We give epidemiological evidence in support of within host lifetime evolution for the first time. A corollary of increased incidence of opportunistic infections is that evolving opportunistic pathogens contribute to the aging process itself both in physiological time and in evolutionary time, and many mechanisms involved in aging are triggered by opportunistic infections (Aguilera et al. 2018; Ulfat et al. 2017). Thus, the cause-effect relationship might actually be inverted. It may not be old age causing infections but the evolving pathogens causing aging. It may not be a coincidence that after the use of antibiotics, which can disturb the course of evolution of the opportunistic pathogens, human life expectancy started increasing dramatically. A more systematic exploration of the evolution of pathogens within the lifetime of a host has the potential not only to understand the mystery of aging but also to improve infection management at a global level.

\section{Acknowledgements}

UB was supported by the Maharashtra Gene Bank Programme during the study period. There was no other funding support for the study.

\section{References}

Aguilera MO, Delgui LR, Romano PS and Colombo MI 2018 Chronic infections: a possible scenario for autophagy and senescence cross-talk. Cells 7162

Bangladesh Bureau of Statistics 2017 Government of the People's Republic of Bangladesh (http://www.bbs.gov.bd/)

Bender BS 2003 Infectious disease risk in the elderly. Immunol. Allergy Clin. North Am. 23 57-64

Boyd R 1995 Basic medical microbiology (Little Brown)

Centers for Disease Control, Prevention (US), National Center for Prevention Services (US). Influenza Vaccine Activity - United States 2012-13 Season and Composition of the 2013-14 (https://www.cdc.gov/mmwr/pdf/wk/ mm6223.pdf)

Centers for Disease Control, Prevention (US), National Center for Prevention Services (US). Prevention and Control of Influenza with Vaccines: Recommendations of the Advisory Committee on Immunization Practices (ACIP) 2010 (https://www.cdc.gov/mmwr/pdf/rr/rr5908. $p d f)$

Chakraborty P 2005 A textbook of microbiology (New Central Book Agency (P) Limited)

Dávila J, Chowell G, Borja-Aburto VH, Viboud C, Grajales Muñiz C and Miller M 2014 Substantial morbidity and mortality associated with pandemic A/H1N1 Influenza in Mexico, winter 2013-2014: Gradual age shift and severity. PLOS Curr. Outbreaks https://doi.org/10.1371/ currents.outbreaks. a855a92f19db1d90ca955f5e908d6631

Dey NC and Dey TK 1973 Medical bacteriology (Allied Agency)

Directorate of Census Operation, Tamil Nadu 2017 (http:// www.census.tn.nic.in/)

Flasche S, Slack M and Miller E 2011 Long term trends introduce a potential bias when evaluating the impact of the pneumococcal conjugate vaccination programme in England and Wales. Euro Surveillance 1619868 
Fulop T, Larbi A, Dupuis G, Le Page A, Frost EH, Cohen AA, Witkowski JM and Franceschi C 2018 Immunosenescence and inflamm-aging as two sides of the same coin: friends or foes? Front. Immunol. 81960

Gardner ID 1980 The effect of aging on susceptibility to infection. Rev. Infect. Dis. 2 801-810

Goering, et al 2012 Mims' medical microbiology (Elsevier)

Goodwin K, Viboud C and Simonsen L 2006 Antibody response to influenza vaccination in the elderly: A quantitative review. In Vaccine 24 1159-1169

Hamilton WD 1980 Sex versus non-sex versus parasite. Oikos 35 282-290

Meyers BR 1989 Infectious diseases in the elderly: an overview. Geriatrics 44 4-6

Pakistan Bureau of Statistics 2017 Population Census (http:// www.pbs.gov.pk/content/population-census)

Papp D, Csermely P and Söti C 2012 A role for SKN-1/Nrf in pathogen resistance and immunosenescence in Caenorhabditis elegans. PLOS Pathog. 8 e1002673

Pawelec G 2018 Age and immunity: what is "immunosenescence"? Expt. Geront. 105 4-9

Ryan, et al 2014 Sherris medical microbiology, Sixth Edition (McGraw Hill Professional)

Schneider EL 1983 Infectious diseases in the elderly. Ann. Intern. Med. 98395

Sidler C, Wóycicki R, Ilnytskyy Y, Metz G, Kovalchuk I and Kovalchuk O 2013 Immunosenescence is associated with altered gene expression and epigenetic regulation in primary and secondary immune organs. Front Genet. 4211
The AIM-HIGH Investigators 2011 Niacin in patients with low hdl cholesterol levels receiving intensive statin therapy. New Engl. J. Med. 365 2255-2267

The NICE-SUGAR Study Investigators 2008 Intensive versus conventional glucose control in critically ill patients. New Engl. J. Med. 360 1283-1297

The Action to Control Cardiovascular Risk in Diabetes Study Group 2008 Effects of intensive glucose lowering in type 2 diabetes. New Engl. J. Med. 358 2545-2559

Tortora GJ, et al 2013 Microbiology: an introduction (Pearson)

Tuberculosis - Respiratory Infections - HPS (http://www. hps.scot.nhs.uk/resp/tuberculosis.aspx\#)

Ulfat B, Akanksha O and Milind W 2017 The evolving opportunistic pathogen communities on host individuals and the evolution of host aging. J. Evol. Med. 5 1-11

Yoshikawa TT 2000 Epidemiology and unique aspects of aging and infectious diseases. Clin. Infect. Dis. 30 931-933

Yoshikawa TT and Norman DC 2017 Geriatric infectious diseases: current concepts on diagnosis and management. J. Am. Geriatr. Soc. 65 631-641

Youngman MJ, Rogers ZN and Kim DH 2011 A decline in p38 MAPK signaling underlies immunosenescence in Caenorhabditis elegans. PLOS Genet. 7 e 1002082

Zerofsky M, Harel E, Silverman N and Tatar M 2005 Aging of the innate immune response in Drosophila melanogaster. Aging Cell 4 103-108

Corresponding editor: NG PRASAD 